\title{
BMJ Open Suitability of emergency department attenders to be assessed in primary care: survey of general practitioner agreement in a random sample of triage records analysed in a service evaluation project
}

\author{
Mary I W Thompson, ${ }^{1}$ Daniel Lasserson, ${ }^{2}$ Lloyd McCann, ${ }^{3}$ Matthew Thompson, ${ }^{2,4}$ \\ Carl Heneghan ${ }^{2}$
}

To cite: Thompson MIW, Lasserson D, McCann L, et al. Suitability of emergency department attenders to be assessed in primary care: survey of general practitioner agreement in a random sample of triage records analysed in a service evaluation project. BMJ Open 2013;3:e003612.

doi:10.1136/bmjopen-2013003612

- Prepublication history for this paper is available online. To view these files please visit the journal online (http://dx.doi.org/10.1136/ bmjopen-2013-003612).

Received 18 July 2013 Revised 28 October 2013 Accepted 1 November 2013

CrossMark

For numbered affiliations see end of article.

Correspondence to Dr Daniel Lasserson; daniel. lasserson@phc.ox.ac.uk

\section{ABSTRACT}

Objectives: To assess the proportion of emergency department (ED) attendances that would be suitable for primary care and the inter-rater reliability of general practitioner (GP) assessment of primary care suitability. Design of study: Survey of GPs' agreement of suitability for primary care on a random anonymised sample of all ED patients attending over a 1-month period.

Setting: ED of a UK Hospital serving a population of 600000.

Method: Four GPs independently used data extracted from clinical notes to rate the appropriateness for management in primary care as well as need for investigations, specialist review or admission. Agreement was assessed using Cohen's $\kappa$.

Results: The mean percentage of patients that GPs considered suitable for primary care management was $43 \%$ (range $38-47 \%$ ). The $\kappa$ for agreement was 0.54 ( $95 \% \mathrm{Cl} 0.44$ to 0.64 ) and $0.47(95 \% \mathrm{Cl} 0.38$ to 0.59$)$. In patients deemed not suitable for primary care, GPs were more likely to determine the need for specialist review (relative risks $(\mathrm{RR})=3.5,95 \% \mathrm{Cl} 3.0$ to $4.2, \mathrm{p}<0.001$ ) and admission (RR=3.9, $95 \% \mathrm{Cl} 3.2$ to $4.7, p<0.001$ ). In patients assessed as suitable for primary care, GPs would initiate investigations in $51 \%$ of cases. Consensus over primary care appropriateness was higher for paediatric than for adult attenders.

Conclusions: A significant number of patients attending ED could be managed by GPs, including those requiring investigations at triage. A stronger agreement among GPS over place of care may be seen for paediatric than for adult attenders. More effective signposting of patients presenting with acute or urgent problems and supporting a greater role for primary care in relieving the severe workflow pressures in ED in the UK are potential solutions.

\section{INTRODUCTION}

There has been a substantial increase in the number of patients attending hospital

\section{Strengths and limitations of this study}

- The sample of records was randomly generated and the general practitioners (GPS) did not know the outcome of the patient attendance.

- The number of GPs was small and the audit was carried out in one healthcare setting, which limits generalisability of our results.

- The definition of acceptability for primary care was made subjectively by the GPs.

emergency departments (EDs) over the last 6 years in England. ${ }^{1}$ The delivery of emergency healthcare in England is under significant threat currently, partly due to rising demand, and improvements to emergency care provision are now a major National Health Service (NHS) priority. ${ }^{2}$ Overcrowding in EDs is associated with delays in initiating treatment, ${ }^{3}$ deficiencies in the processes of care, ${ }^{4}$ a poorer patient experience ${ }^{5}$ and higher mortality in patients who are subsequently admitted. ${ }^{6}$ The introduction of the $4 \mathrm{~h}$ wait limit for patients in EDs in England reduced the average waiting time for treatment and discharge, ${ }^{7}$ which in itself reduces the risk of harm experienced by patients who leave without being seen due to long waits, ${ }^{8}$ but there are substantial pressures on the capacity of ED staff to continue to deliver this standard of care. $^{2}$

The cross-sectional association between less timely access to primary care and greater rates of self-referred discharged ED attendances ${ }^{1}$ implies that a proportion of those attending can be managed in primary care. Although a review of causes of overcrowding in EDs suggests that delay in transfer of admitted patients to a hospital ward is important, ${ }^{9}$ there is evidence that demand can be reduced by increasing access to 
primary care outside normal office hours, from a study in the Netherlands in 2001/2002 using a before and after design. ${ }^{10}$ However, given the multiple reasons for attendance at EDs with non-urgent problems, ${ }^{11-14}$ there is no clear intervention that can be implemented prior to attendance. Therefore, a number of interventions designed for patients who present with non-urgent problems have been trialled within EDs, such as signposting to primary care ${ }^{15}$ or employing primary care physicians (general practitioners; GPs). ${ }^{16}{ }^{17}$

Accurate identification of non-urgent cases in EDs for either redirection to primary care or to be seen within EDs by a GP could improve cost effectiveness of emergency care, ${ }^{18}$ particularly as some studies suggest that non-urgent presentations receive less investigations and follow-up if managed by a GP. ${ }^{19}$ However, there is no consensus over how best to screen for non-urgent presentations at triage. ${ }^{20}$ Nevertheless, estimates of the proportion of patients who attend EDs who could be safely and appropriately managed in primary care are around $30 \%$ for both adults ${ }^{21}$ and children. ${ }^{22}$ Furthermore, in settings where EDs offer direct access to a GP, audits suggest that between $10 \%$ and $30 \%$ of overall attendances can be managed by a GP. ${ }^{23}$

Although incorporating primary care services within EDs offers the potential for improved patient flow and reduced costs, a Cochrane review of primary care services in EDs concluded that the evidence base is currently too weak to make recommendations as only three non-randomised studies were included. ${ }^{24}$ Our aim in this study was to measure the level of agreement among primary care physicians about who could be appropriately managed in primary care, within different groups of patients (based on age range or clinical categories of trauma, medicine or surgery) and to assess whether agreement differed between these groups.

\section{METHODS}

The John Radcliffe Hospital ED is a consultant-led, $24 \mathrm{~h}$ service with full resuscitation facilities without a co-located urgent care centre or nearby walk-in centre. This ED has an annual attendance of 120000 and is the only ED for the city of Oxford and referral centre for the surrounding population of 600000 . We developed a pilot data extraction tool based on that used by Lowy et $a l^{25}$ using information from the initial ED presentation of adult and child patients, including demographics, reason for presentation and triage nurse assessment on an initial 20 cases. Two GPs considered that this information was sufficient to reach decisions about whether the patient appeared to be suitable for management in a primary care setting, and if not whether they would be likely to need further investigations, referral or hospital care.

A random sample generator was used to select cases from ED attenders to the John Radcliffe Hospital in Oxford, UK, each day for a 1-month period, November
2008. All attendances were used as a sampling frame, covering all ages and the $24 \mathrm{~h}$ opening of the department. We extracted data that had been established as satisfactory in the pilot study for GP decision-making from the record documented by a triage nurse, and transferred this to an electronic questionnaire. We identified four primary care physicians, who had been fully accredited for more than 2 years and spent at least $50 \%$ of their professional time in routine general practice, to assess the cases. Two physician pairs each assessed half the sample. Each case was assessed independently by each physician for (1) appropriateness for primary care management; (2) need for investigations; (3) need for specialist review (without admission) and (4) need for hospital admission. Responses were limited to 'yes', 'no' and 'unsure'.

We powered the study to test the hypothesis that the level of agreement between a physician pair was substantial $(\kappa=0.8)$ compared with moderate $(\kappa=0.6)$ based on a probability of $50 \%$ of cases determined appropriate for primary care management. For $\alpha$ of 0.05 , with $80 \%$ power we required a sample which equalled 765 in total.

Raw agreement results are presented as proportions and ranges. Responses were dichotomised to 'yes' versus 'no' and 'unsure', to conservatively estimate those suitable for primary care management. Agreement was assessed using Cohen's $\kappa$ using SPSS (V.17.0) for the overall sample and in clinical subgroups of age (adult vs paediatric), and broad categories of specialties (trauma, medical, surgical). Differences between proportions of primary and non-primary care cases requiring investigations, review or admission were summarised with relative risks (RR) and $\mathrm{z}$ tests used to assess significance of difference.

This study was conducted as a service evaluation and data were obtained primarily for audit purposes according to the guidance from the Oxford Radcliffe Hospital Trust audit policies. In accordance with the guidance for research in place at the time the study was conducted, research ethics approval was not required for service evaluations such as this study. All personnel involved in handling data were employees of the hospital trust or (then) primary care trust. Data were anonymised and treated according to the standard operating procedures for patient data in place at the Trust and the University of Oxford Department of Primary Care.

\section{RESULTS}

Of the 765 case notes retrieved, $629(82 \%)$ contained sufficient information to include in the sample questionnaire (figure 1). Of the total, $25 \%$ were children $(<16$ years old) and $57 \%$ were triaged to the 'minor injuries' area of the ED on initial presentation. From the information presented in the electronic questionnaire, the GPs were able to make a decision on suitable location of management in $88 \%$ of cases $(1017 / 1258$ responses). 


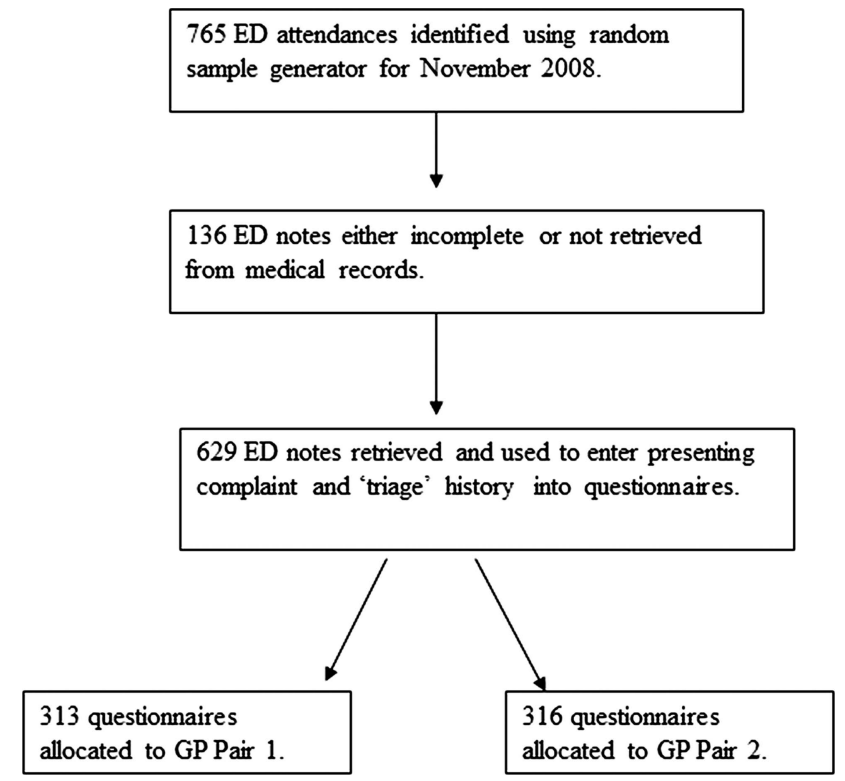

Figure 1 Selection of emergency department case notes reviewed by general practitioners.

The mean percentage of patients that GPs considered suitable for primary care management was $43 \%$ (range $38-47 \%$ ). Table 1 shows that agreement for this initial question for pair 1 gave a $\kappa$ of 0.54 (95\% CI 0.44 to 0.64 ) and for pair 2, 0.47 (95\% CI 0.38 to 0.59$)$.

Figure 2 shows that in cases deemed suitable for primary care management, significantly fewer patients were deemed to require investigations, specialist review or admission than ED cases or where physicians were unsure of the appropriate setting (figure 2). Among patients considered unsuitable for primary care management, GPs were almost four times as likely to determine the need for specialist review ( $80 \%$ vs $23 \%$, RR $=3.5,95 \%$ CI 3.0 to 4.2 , $\mathrm{p}<0.001)$ and for admission $(66 \%$ vs $17 \% \mathrm{RR}=3.9,95 \%$ CI 3.2 to $4.7, \mathrm{p}<0.001$ ) when compared with patients considered suitable for primary care. Patients considered unsuitable for management in primary care were also more likely to need investigations than primary care cases $(86 \%$ vs $51 \%, \mathrm{RR}=1.795 \%$ CI 1.5 to $1.8, \mathrm{p}<0.001)$.

The mean percentage of patients whom GPs considered suitable for primary care management was $42 \%$ in adults (range 36-49\%) and $48 \%$ in children (range $40-57 \%$ ). Table 2 shows that the strength of agreement was consistently higher for children across the clinical categories of trauma ( $\kappa$ 0.62-0.64) and medical and surgical presentations $(\kappa 0.63-0.65)$.

\section{DISCUSSION}

\section{Main findings}

Our results suggest that, on average, 4 out of 10 adults and children attending the ED could potentially be managed in primary care settings. While our study examined potential or likely management rather than actual management, it confirms the need to urgently review the current way of managing acutely unwell adults and children in the NHS. ${ }^{2}$ Differences between cases considered appropriate for primary care compared with those appropriate for the ED were most pronounced over the need for specialist review or admission, rather than the need for investigations. Agreement between GPs over which ED attenders could be managed in primary care is modest, but was higher for children than for adult patients. This superior agreement is preserved across broad categories of clinical presentations as described in presenting triage symptoms and clinical data extracted from the triage histories. In general, similar proportions of adult and paediatric attenders could be seen in primary care, but there appears to be a stronger consensus over the paediatric cases. The fact that agreement among experienced GPs is not high demonstrates that accurate assessment is difficult for individual patients and this needs to be considered in the design of future interventions.

\section{Comparison with the literature}

Our results are similar to a New Zealand study which found equivalent level of agreement among clinicians about which patients could be managed in primary care with a retrospective case notes audit. ${ }^{20}$ They found higher agreement among GPs than among ED specialists over which patients would be appropriate for primary care, but did not explore patient factors that may influence the levels of agreement. Although tools exist to help decide appropriateness for ED care from clinical records, ${ }^{26}$ they have found similar proportions of cases deemed suitable for primary care as those found in our study relying on subjective judgement. ${ }^{26}$ One older study using primary care physicians to rate case notes for primary care appropriateness did not explore the role of patient factors and reported lower levels of agreement $(\kappa=0.34)$, even though raters had access to results of investigations and outcome of ED attendance. ${ }^{27}$ Low levels of agreement among different professionals about appropriateness of different services for patients attending EDs and walk-in-centres have also

Table 1 Levels of agreement between primary care physician pairs, $\kappa(95 \% \mathrm{Cl})$

\begin{tabular}{lllll}
$\kappa(95 \% \mathrm{Cl})$ & $\begin{array}{l}\text { Suitable for primary } \\
\text { care }\end{array}$ & $\begin{array}{l}\text { Further investigations } \\
\text { required }\end{array}$ & $\begin{array}{l}\text { Hospital review } \\
\text { required }\end{array}$ & $\begin{array}{l}\text { Hospital admission } \\
\text { required }\end{array}$ \\
\hline GP pair 1 & $0.54(0.44$ to 0.64$)$ & $0.41(0.31$ to 0.51$)$ & $0.35(0.2$ to 0.51$)$ & $0.22(0.002$ to 0.445$)$ \\
GP pair 2 & $0.47(0.38$ to 0.59$)$ & $0.49(0.39$ to 0.59$)$ & $0.31(0.19$ to 0.43$)$ & $0.48(0.32$ to 0.64$)$ \\
\hline GP, general practitioner. & & &
\end{tabular}




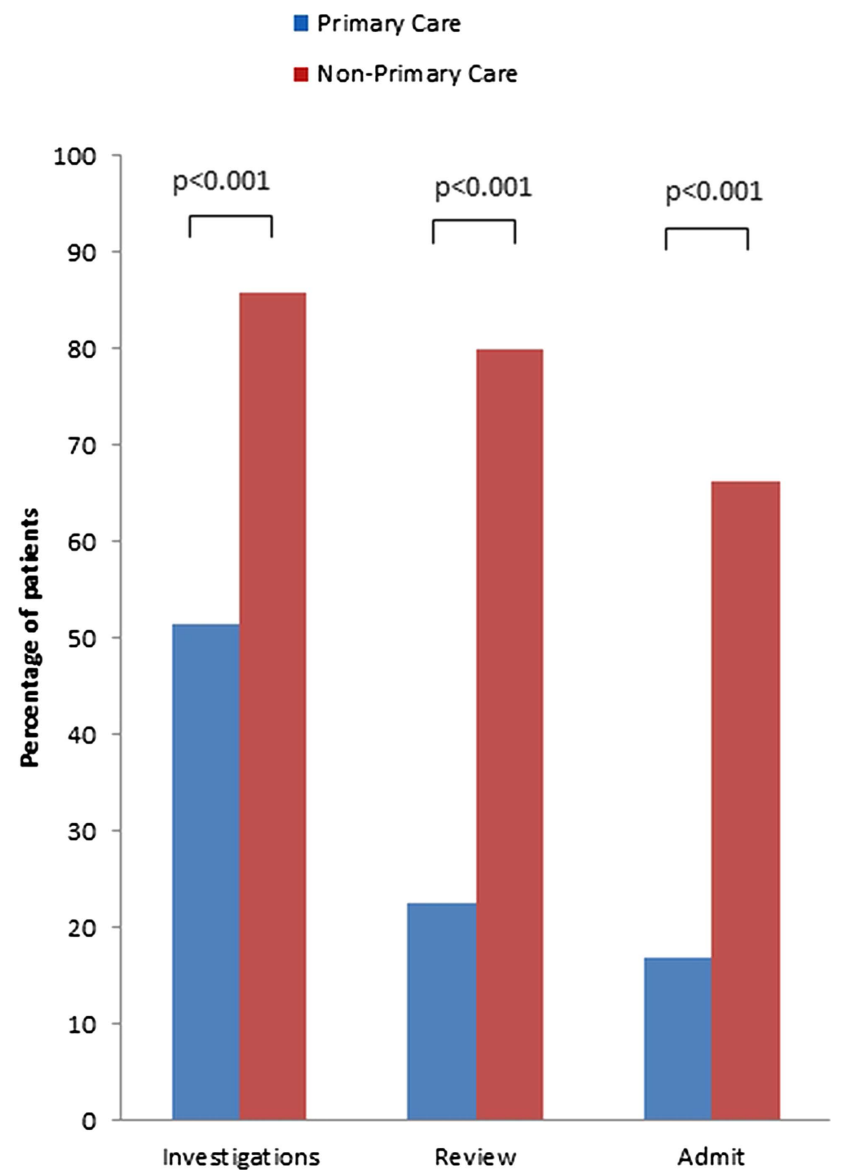

Figure 2 Percentage of patients considered suitable for primary care versus not suitable for primary requiring investigations, specialist review or hospital admission.

been reported, but may be explained by the fact that different professional groups were used to determine consensus. ${ }^{28}$

The weak evidence base to inform urgent care service redesign has previously been highlighted in the Primary Care Foundation's report ${ }^{23}$ and is confirmed in a recent Cochrane review. ${ }^{24}$ The triage model employed to appropriately select primary care patients among the incoming ED workstream is likely to be a critical factor in the success of introducing primary care services for patients who have already presented to an ED. However, there is evidence that, in general, triage of ED patients varies depending on the individual assessing patients, even if formal triage systems are used. ${ }^{29} 30$ One alternative to selecting patients after ED attendance via triage is to co-locate primary care services near EDs which enables patients to self-select for urgent primary care rather than attending the $\mathrm{ED}$ as it becomes easier to choose which service they feel is most appropriate, given that they have made the decision to seek healthcare urgently. In an uncontrolled comparison, providing an out-of-hours primary care service near the ED in Maastricht resulted in 35\% fewer ED attenders than another ED without similar primary care provision. ${ }^{31}$

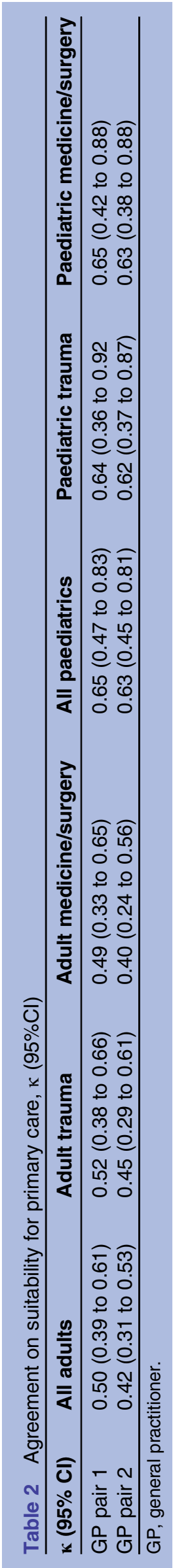




\section{Strengths and limitations}

The strengths of this study are that information on investigations undertaken and clinical outcomes was not provided, which reduces bias in the assessment of primary care appropriateness. Our findings, therefore, reflect the more realistic situation of a patient attending ED where this information is obviously not yet known. Our sample was random and included all ages, clinical presentations and severity of illness, implying that our results are generalisable to the overall ED workstream. Nevertheless our study has some limitations. First, we do not have follow-up data to determine the true level of agreement between GP assessment and subsequent clinical care, including, for example, the proportion of those considered appropriate for primary care who were sent home from ED or the proportion of those considered as needing admission who were admitted. Second, the sample of GPs was small and their individual clinical areas of expertise and comfort with different clinical presentations may not be representative of all primary care physicians, and the definition of acceptability for primary care was left to individual judgement. This may be reflected in the modest levels of agreement that we found. Third, clinical data extracted from triage histories were incomplete in some cases which may have contributed to further variation in responses. Finally we did not ask the GPs whether they considered other community-based health professionals, for example, pharmacists, as an appropriate choice of healthcare access given the clinical presentations. In addition, this study was carried out in one university hospital during 1 month of the year which may not generalise to other settings. Given the importance of these results, we suggest that they are replicated in other settings to test generalisability.

\section{Clinical and policy implications}

Our results add to growing concerns that an increasing number of patients attending ED services in the UK threaten not only patient care but also efforts to contain healthcare spending. Even if the true proportion of adults and children currently seen in ED who could be seen in primary care is less than the $43 \%$ that our study estimated, it implies that a major restructuring of how urgent or emergency care is provided is urgently needed. This needs to include ways to potentially signpost patients more effectively, while simultaneously providing support for providing urgent primary care service (when daytime primary care is already under severe pressure). Importantly, the need for investigations was not seen to be a barrier to primary care assessment. Generating consensus criteria to identify patients who can be managed in primary care, implementing these criteria and measuring the effects on patient flow, healthcare costs and patient satisfaction are the major priorities.

\author{
Author affiliations \\ ${ }^{1}$ Department of Epidemiology and Public Health, University of Exeter Medical \\ School, Exeter, UK
}

${ }^{2}$ Department of Primary Care Health Sciences, University of Oxford, Oxford, UK

${ }^{3}$ Medical Services, MercyAscot Hospitals, Auckland, New Zealand ${ }^{4}$ Department of Family Medicine, University of Washington, Seattle, Washington, USA

Acknowledgements The authors would like to thank Dr Ele Lambert, Dr Merlin Wilcox, Dr Joe McManners, Dr Michael Moher, Dr Andy Chivers and Dr Simon Plint.

\section{Collaborators Ele Lambert.}

Contributors MIWT, LMcC and EL conceived the study and collected the data. $\mathrm{CH}$ and MT designed the study. DSL contributed data, analysed it and drafted the manuscript. All authors contributed to data interpretation, manuscript revisions and agreed on the final manuscript.

Funding A small grant was provided by the NHS Oxfordshire to four primary care physicians to conduct the audit. DL is supported by the NIHR Oxford Biomedical Research Centre and the Department of Primary Care Health Sciences and $\mathrm{CH}$ is supported by the NIHR School for Primary Care Research.

\section{Competing interests None.}

Ethics approval This study was conducted as a service evaluation and data were obtained primarily for audit purposes according to the guidance from the Oxford Radcliffe Hospital Trust audit policies. In accordance with the guidance for research in place at the time the study was conducted, research ethics approval was not required for service evaluations such as this study. All personnel involved in handling data were employees of the hospital trust or (then) primary care trust. Data were anonymised and treated according to the standard operating procedures for patient data in place at the Trust and the University of Oxford Department of Primary Care.

Provenance and peer review Not commissioned; externally peer reviewed.

Data sharing statement The data on which this analysis is based are available electronically from the corresponding author.

Open Access This is an Open Access article distributed in accordance with the Creative Commons Attribution Non Commercial (CC BY-NC 3.0) license, which permits others to distribute, remix, adapt, build upon this work noncommercially, and license their derivative works on different terms, provided the original work is properly cited and the use is non-commercial. See: http:// creativecommons.org/licenses/by-nc/3.0/

\section{REFERENCES}

1. Cowling TE, Cecil EV, Soljak MA, et al. Access to primary care and visits to emergency departments in England: a cross-sectional, population-based study. PLOS ONE 2013;8:e66699.

2. NHS England. Urgent and emergency care review-evidence base engagement document. London: Department of Health, 2013.

3. Mills AM, Shofer FS, Chen EH, et al. The association between emergency department crowding and analgesia administration in acute abdominal pain patients. Acad Emerg Med 2009;16:603-8.

4. Lee CC, Lee NY, Chuang MC, et al. The impact of overcrowding on the bacterial contamination of blood cultures in the ED. Am J Emerg Med 2012;30:839-45

5. Collis J. Adverse effects of overcrowding on patient experience and care. Emerg Nurse 2010;18:34-9.

6. Sun BC, Hsia RY, Weiss RE, et al. Effect of emergency department crowding on outcomes of admitted patients. Ann Emerg Med 2013;61:605-11 e6.

7. Mason S, Weber EJ, Coster J, et al. Time patients spend in the emergency department: England's 4-hour rule-a case of hitting the target but missing the point? Ann Emerg Med 2012;59:341-9.

8. $\mathrm{Ng} \mathrm{Y}$, Lewena S. Leaving the paediatric emergency department without being seen: understanding the patient and the risks. $J$ Paediatr Child Health 2012;48:10-15.

9. Hoot NR, Aronsky D. Systematic review of emergency department crowding: causes, effects, and solutions. Ann Emerg Med 2008;52:126-36.

10. van Uden CJ, Crebolder HF. Does setting up out of hours primary care cooperatives outside a hospital reduce demand for emergency care? Emerg Med J 2004;21:722-3. 
11. Agarwal S, Banerjee J, Baker R, et al. Potentially avoidable emergency department attendance: interview study of patients' reasons for attendance. Emerg Med J 2012;29:e3.

12. Benahmed N, Laokri S, Zhang WH, et al. Determinants of nonurgent use of the emergency department for pediatric patients in 12 hospitals in Belgium. Eur J Pediatr 2012;171:1829-37.

13. Philips $\mathrm{H}$, Remmen $\mathrm{R}$, De Paepe $\mathrm{P}$, et al. Out of hours care: a profile analysis of patients attending the emergency department and the general practitioner on call. BMC Fam Pract 2010;11:88.

14. Koziol-McLain J, Price DW, Weiss B, et al. Seeking care for nonurgent medical conditions in the emergency department: through the eyes of the patient. J Emerg Nurs 2000;26:554-63.

15. Gentile $S$, Vignally P, Durand AC, et al. Nonurgent patients in the emergency department? A French formula to prevent misuse. BMC Health Serv Res 2010;10:66.

16. Bury $G$, Hungerford $P$, Langton $D$, et al. A \& $E$ services in Ireland: the potential role of general practice in accident and emergency services. Ir J Med Sci 2000;169:245-7.

17. Kool RB, Homberg DJ, Kamphuis HC. Towards integration of general practitioner posts and accident and emergency departments: a case study of two integrated emergency posts in the Netherlands. BMC Health Serv Res 2008;8:225.

18. Lee $A$, Hazlett $C B$, Chow $S$, et al. How to minimize inappropriate utilization of Accident and Emergency Departments: improve the validity of classifying the general practice cases amongst the A\&E attendees. Health Policy 2003;66:159-68.

19. Huibers L, Thijssen W, Koetsenruijter J, et al. GP cooperative and emergency department: an exploration of patient flows. J Eval Clin Pract 2013;19:243-9.

20. Elley CR, Randall PJ, Bratt D, et al. Can primary care patients be identified within an emergency department workload? N Z Med J 2007;120:U2583
21. Dale J, Green J, Reid F, et al. Primary care in the accident and emergency department: I. Prospective identification of patients. BMJ 1995;311:423-6.

22. Pileggi C, Raffaele G, Angelillo IF. Paediatric utilization of an emergency department in Italy. Eur J Public Health 2006;16:565-9.

23. Carson D, Stern $\mathrm{R}$, Clay $\mathrm{H}$. Urgent care. A practical guide to transforming same-day care in general practice. Lewes, East Sussex: Primary Care Foundation, 2009.

24. Khangura JK, Flodgren G, Perera R, et al. Primary care professionals providing non-urgent care in hospital emergency departments. Cochrane Database Syst Rev 2012;(11):CD002097.

25. Lowy A, Kohler B, Nicholl J. Attendance at accident and emergency departments: unnecessary or inappropriate? J Public Health Med 1994;16:134-40.

26. Sempere-Selva T, Peiro S, Sendra-Pina P, et al. Inappropriate use of an accident and emergency department: magnitude, associated factors, and reasons-an approach with explicit criteria. Ann Emerg Med 2001;37:568-79.

27. Gribben B. General practitioners' assessments of the primary care caseload in Middlemore Hospital Emergency Department. N Z Med J 2003;116:U329

28. Bickerton J, Davies J, Davies $\mathrm{H}$, et al. Streaming primary urgent care: a prospective approach. Prim Health Care Res Dev 2012;13:142-52.

29. Dallaire C, Poitras J, Aubin K, et al. Emergency department triage: do experienced nurses agree on triage scores? J Emerg Med 2012;42:736-40.

30. Nakagawa J, Ouk S, Schwartz B, et al. Interobserver agreement in emergency department triage. Ann Emerg Med 2003:41:191-5.

31. van Uden CJ, Winkens RA, Wesseling G, et al. The impact of a primary care physician cooperative on the caseload of an emergency department: the Maastricht integrated out-of-hours service. J Gen Intern Med 2005;20:612-17. 